\title{
Erectile function after kidney transplantation: a meta-analysis
}

\author{
Jiaqi Kang, Jia Tian, Yi Lu, Yuxuan Song, Xiaoqiang Liu \\ Department of Urology, Tianjin Medical University General Hospital, Tianjin, China \\ Contributions: (I) Conception and design: J Kang, X Liu. (II) Administrative support: None. (III) Provision of study material or patients: None. (IV) \\ Collection and assembly of data: J Kang, J Tian. (V) Data analysis and interpretation: J Kang, Y Lu, Y Song. (VI) Manuscript writing: All authors. (VII) \\ Final approval of manuscript: All authors. \\ Correspondence to: Xiaoqiang Liu, PhD. Department of Urology, Tianjin Medical University General Hospital, Heping District, 154 Anshan Road, \\ Tianjin 300052, China. Email: waikebenniao@163.com.
}

Background: Erectile dysfunction (ED) is common in patients with end-stage renal disease (ESRD). Whether kidney transplantation can improve erectile function in patients with ESRD is still controversial. We conducted a meta-analysis on the relationship between kidney transplantation and erectile function.

Methods: A literature search was conducted on PubMed, Embase, Cochrane Library, and Web of Science until May 31, 2019. Primary outcomes were ED prevalence and each domain score of the International Index of Erectile Function (IIEF) questionnaire. We used age-matched dialysis patients or patients before kidney transplantation as a control group and compared them to kidney transplant recipients.

Results: A total of 9 articles were finally enrolled in the study. Compared with the control group, the kidney transplantation group had a lower prevalence of ED (OR 0.49, 95\% CI: 0.28-0.86) and higher domain scores for erectile function (SMD 0.53, 95\% CI: 0.12-0.94) and sexual desire (SMD 1.19, 95\% CI: 0.11-2.27). While there were no significant variations in domain scores for orgasmic function (SMD 0.27, 95\% CI: -0.10-0.63), intercourse satisfaction (SMD 0.26, 95\% CI: -0.10-0.61), and overall satisfaction (SMD $0.17,95 \%$ CI: -0.21-0.56). Patients in the kidney transplantation group had higher serum testosterone (SMD 1.20, 95\% CI: 0.86-1.54) and lower prolactin (SMD -1.46, 95\% CI: -2.22 to -0.69) and luteinizing hormone (SMD $-0.97,95 \%$ CI: -1.39 to -0.55$)$.

Conclusions: Kidney transplantation may be associated with improved erectile function in patients with ESRD. This may be attributable to the correction of endocrine hormone disorders in patients after kidney transplantation.

Keywords: Erectile dysfunction (ED); end-stage renal disease (ESRD); kidney transplantation; meta-analysis

Submitted Feb 19, 2020. Accepted for publication Aug 28, 2020.

doi: 10.21037/tau-20-604

View this article at: http://dx.doi.org/10.21037/tau-20-604

\section{Introduction}

Erectile dysfunction (ED) is defined as the persistent inability to attain and maintain an erection sufficient to permit satisfactory sexual performance (1), which may affect physical and psychosocial health and have a significant influence on the quality of life (QoL) of sufferers and their partner's (2). ED is a major sequela of end-stage renal disease (ESRD) that is often overlooked by providers. The metabolic, homeostatic, hormonal, cardiovascular, and neurologic physiology of ESRD can all lead to ED (3). A new study has reported that the prevalence of ED in ESRD patients, especially those on hemodialysis, is as high as $84 \%$, which is higher than in the general population (4).

Kidney transplantation has been widely recognized as the most effective treatment for ESRD. With the popularity and maturity of transplantation technology, the number of patients with ESRD receiving kidney transplantation has increased year by year. And the survival time of kidney transplantation recipients has significantly prolonged. Therefore, the QoL of kidney transplantation recipients, especially the erectile function of male recipients, has 
attracted more and more attention from the doctors and transplant patients. However, the role of kidney transplantation on erectile function in patients with ESRD is still questionable.

Therefore, we performed a meta-analysis of available literature to assess the impact of kidney transplantation on erectile function in patients with ESRD.

We present the following article in accordance with the PRISMA reporting checklist (available at http://dx.doi. org/10.21037/tau-20-604).

\section{Methods}

\section{Search strategy}

The study is reported according to the Preferred Reporting Items for Systematic Reviews and Meta-Analyses (PRISMA) Statement. The study protocol was registered on PROSPERO (registration number CRD42020163633). Two authors (KANG JQ, TIAN J) independently searched relevant literature on PubMed, Embase, Cochrane Library, and Web of Science, published before May 31, 2019. The retrieval strategy we used for PubMed is as follows: ((((("Penile Erection"[Mesh]) OR ((erectile function) OR sexual function)) OR "Sexual Behavior"[Mesh])) AND ((((()uremia) OR "Uremia"[Mesh])) OR ((Dialysis[MeSH Terms]) OR dialyses $))$ OR $((((()(((($ dialyses, renal) OR renal dialysis) OR dialysis, renal) OR hemodialysis) OR hemodialyses) OR dialysis, extracorporeal) OR dialyses, extracorporeal) OR extracorporeal dialyses) OR extracorporeal dialysis)) OR "Renal Dialysis"[Mesh])) OR

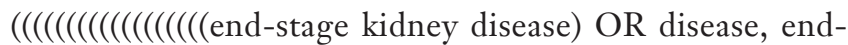
stage kidney) OR end stage kidney disease) OR kidney disease, end-stage) OR chronic kidney failure) OR endstage renal disease) OR disease, end-stage renal) OR end stage renal disease) OR renal disease, end-stage) OR renal disease, end stage) OR renal failure, end-stage) OR endstage renal failure) OR renal failure, end stage) OR renal failure, chronic) OR chronic renal failure) $\mathrm{OR}$ esrd)) $\mathrm{OR}$ "Kidney Failure, Chronic"[Mesh]))) AND (("Kidney Transplantation"[Mesh]) OR (((()(((renal transplantation) OR renal transplantations) OR transplantations, renal) OR transplantation, renal) OR grafting, kidney) OR kidney grafting) OR transplantation, kidney) OR kidney transplantations) OR transplantations, kidney)). We adjusted the keywords when searching literature on other databases. Besides, references of retrieved literature were also screened to acquire extra eligible studies.

\section{Inclusion criteria}

Studies meeting the following criteria were included: (I) the study evaluated the relationship between kidney transplantation and erectile function with no limitation about study design; (II) Erectile function is evaluated by the International Index of Erectile Function (IIEF) questionnaire; (III) Control groups were age-matched dialysis patients or patients before kidney transplantation; (IV) sufficient data to calculate the odds ratios (ORs) or weighted mean differences (WMDs) with 95\% confidence intervals (CIs). Studies with insufficient data were excluded.

\section{Data extraction}

The two authors (KANG JQ and TIAN J) extracted the data from included studies independently using a standardized form. When the two disagreed, a third author (LIU XQ) would participate in the discussion to make a decision. The data we extracted includes: first author, publication year, study design, sample size, ethnicity, and the major outcomes, like erectile function and hormone levels.

The IIEF-15 is a 15 -item questionnaire evaluating five domains of male sexual function (5): erectile function (EF), sexual desire (SD), intercourse satisfaction (IS), orgasmic function (OF), and overall satisfaction (OS). The questionnaire reflects the ability to achieve sufficient erections and the maintenance of erections. As a short version of IIEF-15, the IIEF-5 containing 5-item does not assess these measures and is only used to assess the erectile function of patients. Patients whose IIEF- 5 score is $\leq 21$, or EF domain score of IIEF- 15 is $\leq 25$ were diagnosed as ED.

\section{Quality evaluation}

We used the 9-point Newcastle-Ottawa Scale (NOS) to assess the quality of included prospective studies (6), from three aspects, including selection, comparability, and outcomes. A total score of 7-9 is defined as high quality, 4-6 is moderate quality, $1-3$ is low quality. Besides, an 11-item scale recommended by the Agency for Healthcare Research and Quality (AHRQ) was used to assess the quality of included cross-sectional studies. An item would be scored "0" if it was answered "No" or "Unclear"; conversely, if it was answered "Yes", then the item would be scored "1". Article quality was estimated as follows: a total score of $0-3$ is defined as low quality, $4-7$ is moderate quality, $8-11$ is high quality. 


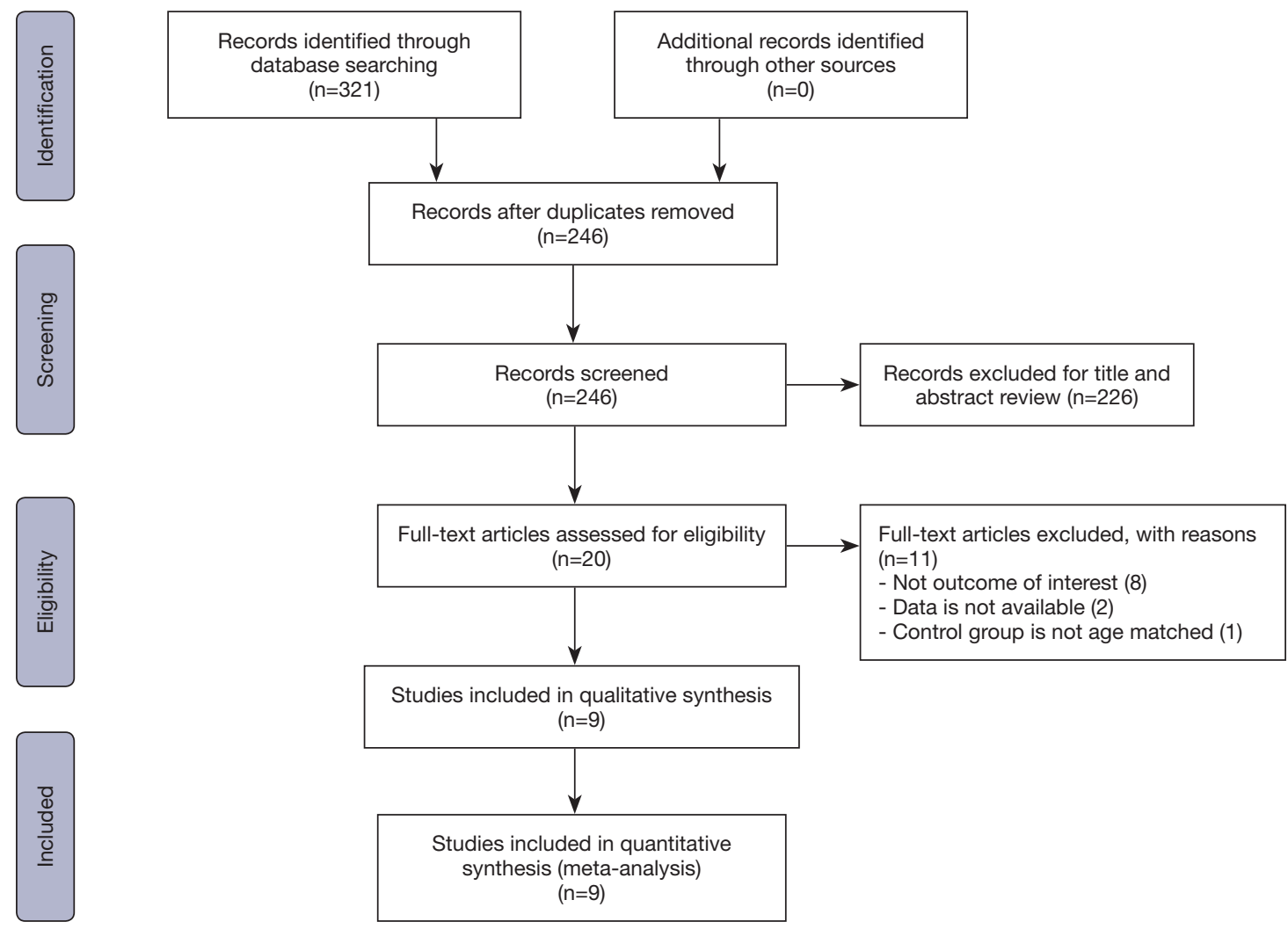

Figure 1 Flowchart of literature screening and selection.

\section{Statistical analysis}

For the prevalence of erectile dysfunction, we chose the Odds ratios (OR) as the effect size and calculated the $95 \%$ confidence interval (95\% CI). For the IIEF scores and hormone levels, we used the standard mean difference (SMD) as the effect size and calculated the 95\% CI. The heterogeneity test among these studies was performed by $\mathrm{I}^{2}$. We made a subgroup analysis when the value of $\mathrm{I}^{2}>50 \%$. Egger's test and Begg's test was used to evaluate publication bias, and there is a significant bias when the $\mathrm{P}<0.05$. To assess the stability and effect of enrolled studies, we performed a sensitivity analysis. All statistical tests for the meta-analysis were performed by Stata version 12.0 (Stata Corporation, College Station, TX, USA).

\section{Results}

\section{Eligible studies}

We retrieved 321 related articles. After removing duplicates, 246 articles are remaining. Further, by reading the title and abstract, we removed 226 articles. We read the full text of the remaining 20 articles. In this study, the main outcomes we were interested in were ED prevalence, IIEF domain scores (including EF, SD, OF, IS, and OS). 8 articles that did not include the above outcomes were excluded by a reason of non-interesting outcome. Two articles were excluded owing to the unavailable data, and 1 article was excluded because the control group was not age-matched. Finally, 9 articles were included in the present meta-analysis (7-15). We adapted a PRISMA flowchart to show the study selection process, which is shown in Figure 1. Of these 9 studies, six prospective studies compared erectile function before and after kidney transplantation in patients with ESRD. The remaining three are cross-sectional studies comparing kidney transplant recipients to age-matched dialysis patients. The characteristics of the included studies were demonstrated in Table 1. All enrolled studies were estimated to be of high-quality according to the NOS and AHRQ scales. The detailed quality scores of each literature were provided in Tables $S 1$ and $S 2$. 
Table 1 Characteristics of included studies

\begin{tabular}{|c|c|c|c|c|c|c|}
\hline Study & Country & Ethnicity & Study design & Sample size & ED evaluation tool & Follow-up period (mo) \\
\hline Mehrsai (2006) & Iran & Caucasian & Prospective study & $64 / 64$ & IIEF-5 & 6 \\
\hline Peng (2007) & China & Asian & Prospective study & $55 / 55$ & IIEF-5 & 9 \\
\hline Mirone (2009) & Italy & Caucasian & Prospective study & $78 / 78$ & IIEF-15 & 12 \\
\hline ال (2010) & China & Asian & Prospective study & $33 / 33$ & IIEF-15 & 6 \\
\hline Teng (2011) & China & Asian & Prospective study & $24 / 24$ & IIEF-5 & 6 \\
\hline Park (2013) & Republic of Korea & Asian & Cross-sectional Study & $25 / 37$ & IIEF-15 & $>11$ \\
\hline Yavuz (2013) & Turkey & Caucasian & Cross-sectional Study & $53 / 47$ & IIEF-5 & $>12$ \\
\hline
\end{tabular}

\section{ED prevalence and erectile function domain score}

In this study, 5 articles used IIEF-15 to evaluate patients' sexual function, and 4 studies used IIEF-5. IIEF-5 and the EF domain score of IIEF-15 (IIEF-EF) are both tools to evaluate patients' erectile function, so they were combined for analysis in the study. As shown in Figure 2, the prevalence of ED was lower in the kidney transplantation group than in the control group (OR 0.49, 95\% CI: 0.28-0.86). Similarly, the EF domain score of the kidney transplantation group was higher than was those of the control group (SMD 0.53, 95\% CI: 0.12-0.94). To decrease the potential bias introduced by the three studies comparing age-matched dialysis patients to transplanted patients, we performed a sub-analysis on the studies with pre- and post- transplanted patients only. As shown in Figure 3, the prevalence of ED was lower in the pre-transplantation group than in the post-transplantation group (OR 0.45, 95\% CI: 0.21-0.98). While no significant difference in the EF domain score was found between pre-transplantation and post-transplantation patients (SMD 0.53, 95\% CI: $-0.03-1.08)$.

\section{The other domain scores of IIEF}

We conducted a meta-analysis of five studies using IIEF-15 to learn about whether kidney transplantation has an impact on other aspects of sexual function. As shown in Figure 4, IIEF-SD score was found to be higher in the kidney transplantation group than in the control group (SMD 1.19, 95\% CI: 0.11-2.27), while there was no statistical difference between the two groups in the other domain scores of IIEF, including IIEF-OF (SMD 0.27, 95\% CI: -0.10-0.63), IIEFIS (SMD 0.26, 95\% CI: -0.10-0.61), and IIEF-OS (SMD $0.17,95 \%$ CI: $-0.21-0.56$ ).

\section{Endocrine hormone levels}

Among all included studies, 3 studies reported changes in testosterone (T) level, and 2 studies reported prolactin (PRL), follicle-stimulating hormone (FSH), and luteinizing hormone (LH). As shown in Figure 5, the testosterone level in the kidney transplantation group was significantly higher than in the control group (SMD 1.20, 95\% CI: 0.86-1.54). On the contrary, lower PRL level (SMD -1.46, 95\% CI: -2.22 to -0.69 ) and LH level (SMD $-0.97,95 \%$ CI: -1.39 to -0.55$)$ were found in the kidney transplantation group. There was no statistical difference in FSH level between the two groups (SMD 0.10, 95\% CI: -0.30-0.50).

\section{Subgroup analysis of erectile function}

We performed a subgroup analysis of ED prevalence and EF domain score that we were most concerned about, due to a large heterogeneity $\left(\mathrm{I}^{2}=65 \%, \mathrm{P}=0.004 ; \mathrm{I}^{2}=87.6 \%, \mathrm{P}=0.000\right)$. Limited to the data provided by the included studies, we performed a subgroup analysis based on the erectile function assessment tool and ethnicity. As shown in Figure 6 , subgroup analysis according to the erectile function assessment tool showed that there was no significant difference in ED prevalence (OR 0.79, 95\% CI: 0.42-1.50, $\mathrm{I}^{2}=48.8 \%$ ) and EF domain score (SMD 0.14, $95 \%$ CI: $-0.28-0.57, \mathrm{I}^{2}=78 \%$ ) between the kidney transplantation group and the control group when IIEF-EF was used to 


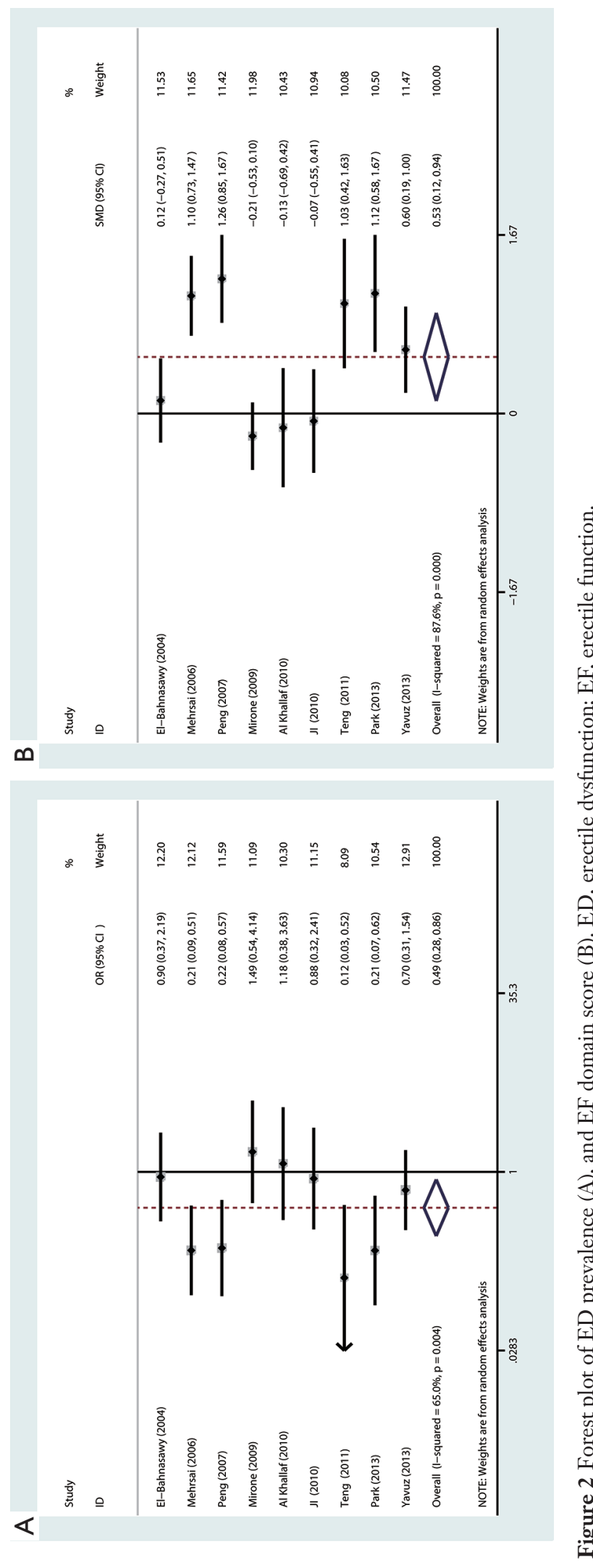



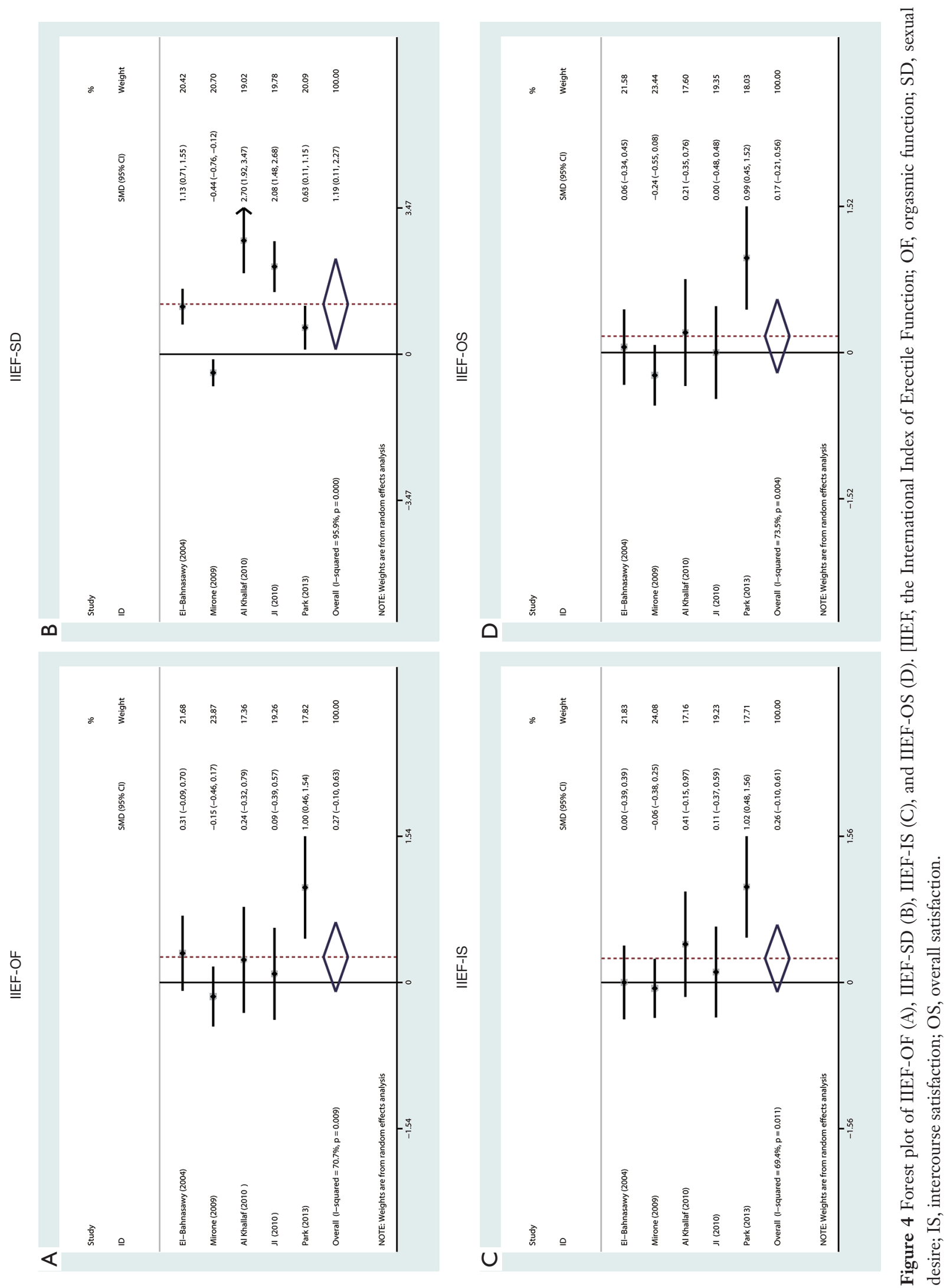

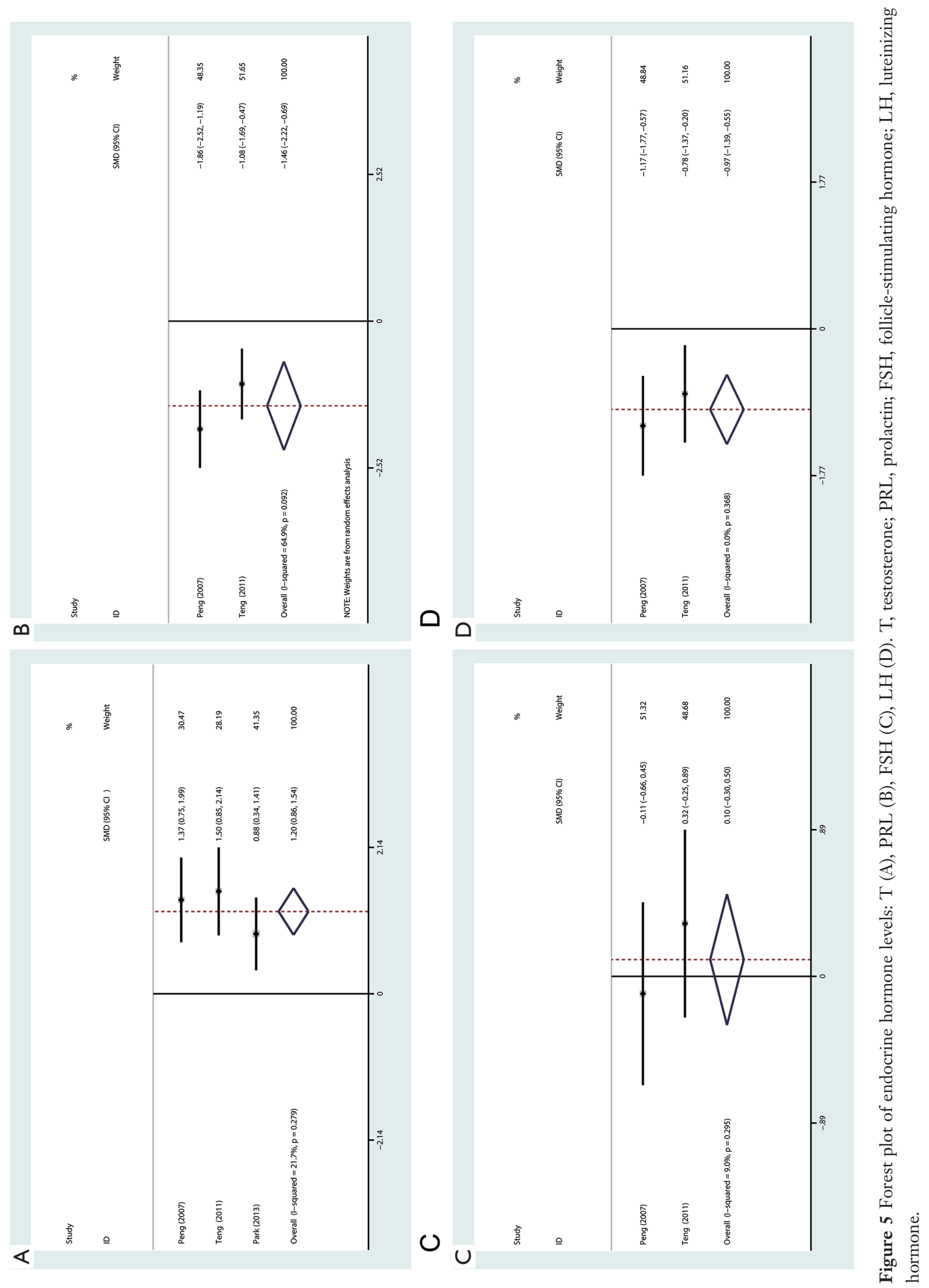

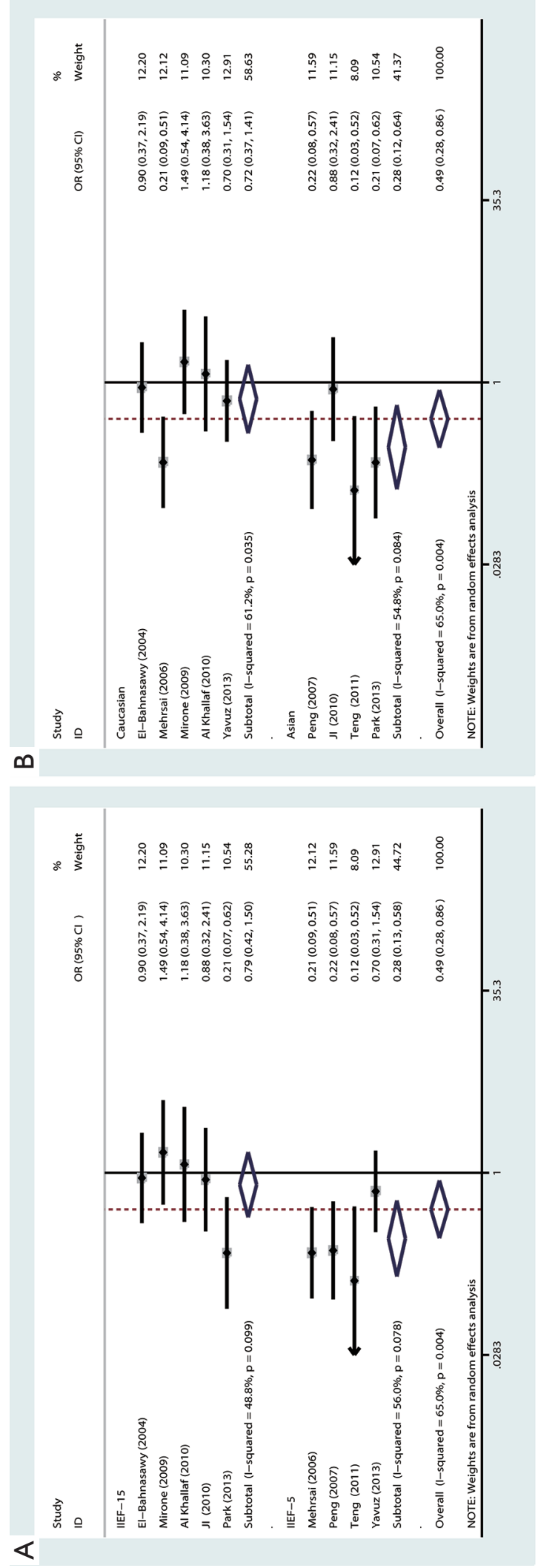

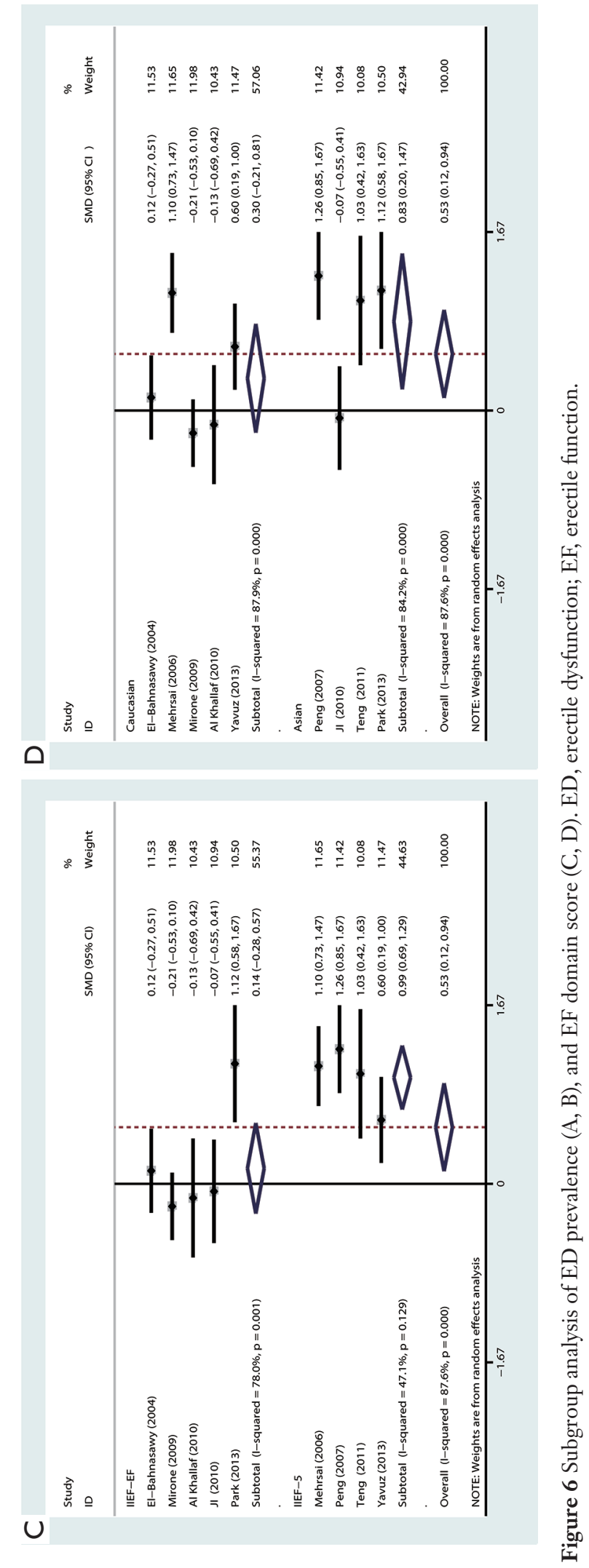

Transl Androl Urol 2020;9(5):1967-1979 I http://dx.doi.org/10.21037/tau-20-604 


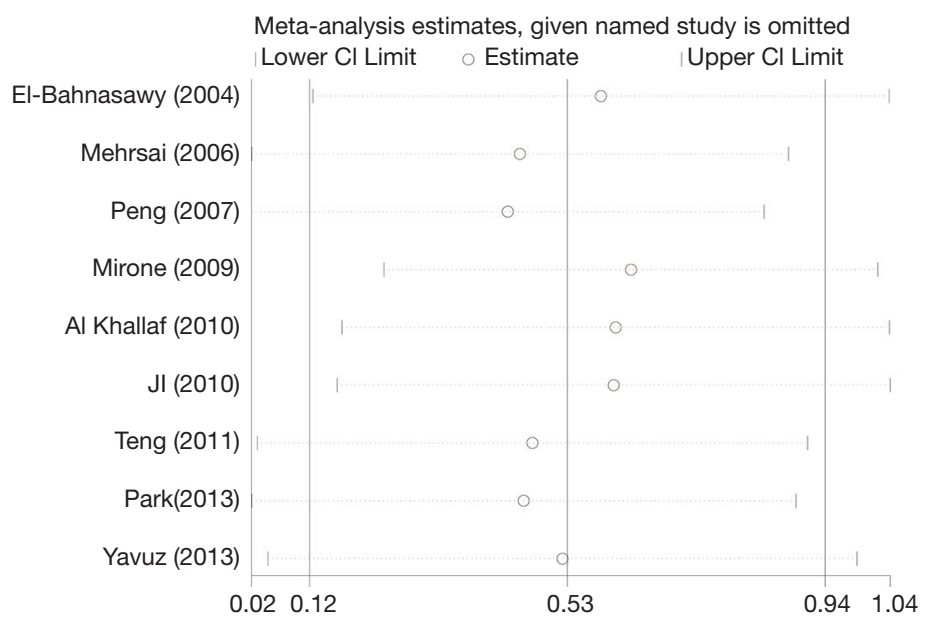

Figure 7 Sensitivity analysis of EF domain score. EF, erectile function.
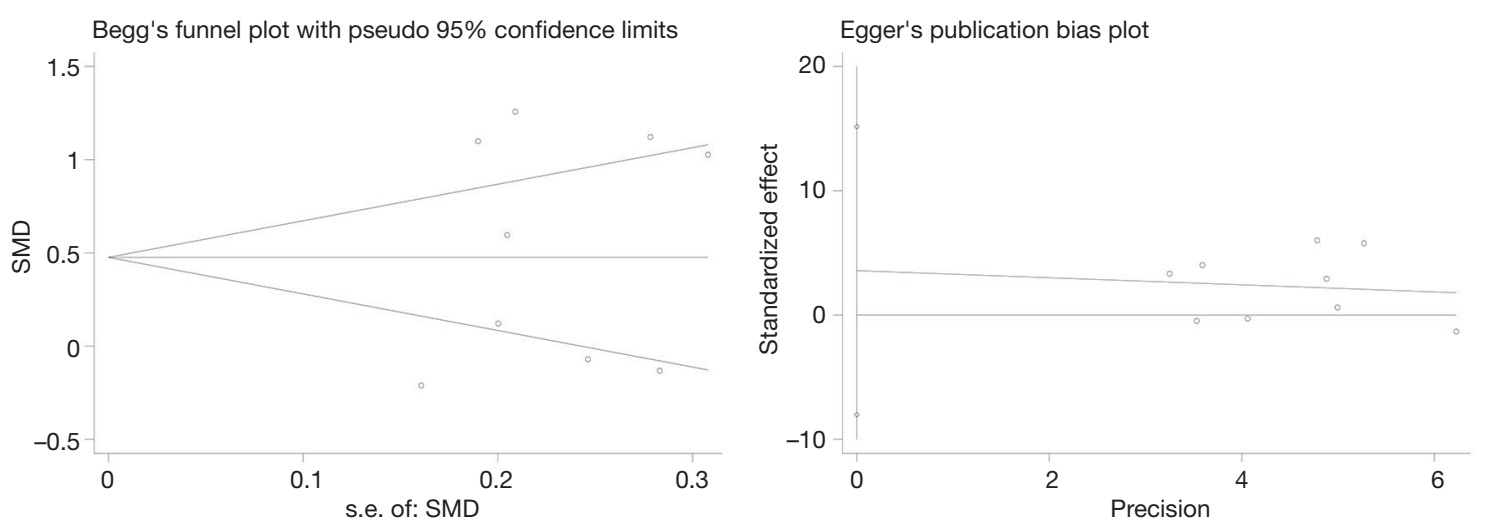

Figure 8 Begg's funnel plot and Egger's publication bias plot of EF domain score. EF, erectile function.

evaluate erectile function. When using IIEF-5, the kidney transplantation group had a lower ED prevalence (OR 0.28, 95\% CI: $0.13-0.58, \mathrm{I}^{2}=56 \%$ ) and higher EF domain score (SMD 0.99, 95\% CI: $0.69-1.29, \mathrm{I}^{2}=47.1 \%$ ) than the control group. Besides, a meta-analysis of Asian studies found that the kidney transplantation group had a lower ED prevalence (OR 0.28 , 95\% CI: $0.12-0.64, \mathrm{I}^{2}=54.8 \%$ ) and higher EF domain score (SMD 0.83, 95\% CI: $0.20-1.47, \mathrm{I}^{2}=84.2 \%$ ) than the control group, as reported in Figure 6. However, no significant difference was found among Caucasians.

\section{Sensitivity analysis}

We performed a sensitivity analysis by removing each enrolled single study in turn to evaluate the influence of an individual study on the pooled results. As shown in Figure 7, our results were very reliable, and no individual study significantly affected the pooled SMD comparing of the EF domain score.

\section{Publication bias}

Begg's and Egger's tests were conducted to evaluate the publication bias of this meta-analysis. As shown in Figure 8 , there was no obvious publication bias concerning the EF domain score, with the $\mathrm{P}$ value being 0.489 .

\section{Discussion}

With the rising prevalence of ESRD, over 100,000 Americans are currently listed for kidney transplantation. Approximately 13,000 patients received a deceased donor 
organ in 2016, and half of these recipients had been on dialysis for $>5$ years (16). And ED is more frequent in patients with chronic renal disease (CKD) (17). As early as 1973, Levy et al. found varying degrees of ED in patients with chronic renal insufficiency (18). It was shown that ED occurred in $82.7 \%$ of CKD patients just entering the dialysis treatment (19), 51.9-88\% of peritoneal dialysis (PD) patients $(20,21)$, and in up to $87.5 \%$ of hemodialysis (HD) patients $(8,22)$. The cause of the high prevalence of ED in patients with ESRD may be related to both the underlying etiology of CKD (e.g., diabetes or hypertension) as well as the direct effects of uremia, particularly on the nervous system (23). Psychogenic ED in the setting of high levels of anxiety and depression, decreased activity, and poor body image associated with patients initiating dialysis is likely also a key driver in this phenomenon (24). ED profoundly influences the quality of life in patients with CKD, which should attract our attention.

It is of importance to acknowledge that the dramatic effect of kidney transplantation on ED is now well documented. Studies have shown that erectile function can be improved in patients receiving kidney transplantation (25), but some scholars consider that there is no significant difference in erectile function between uremic patients who receive kidney transplantation and hemodialysis $(10,11)$. Whether kidney transplantation can improve erectile function in patients with ESRD is still controversial.

Therefore, we did the first meta-analysis to compare the erectile function before and after kidney transplantation. Our results showed that kidney transplant recipients had a lower prevalence of ED and a higher erectile function score. That is to say, receiving kidney transplantation might help improve the erectile function of ESRD patients. Consistent with our meta-analysis, Ahmad et al. found that there was improvement in $40 \%$ of patients in the erectile functions after 6 months of kidney transplantation (26). Based on limited data (ethnicity and evaluation tools), we conducted a subgroup analysis to look for sources of heterogeneity. In a subgroup analysis based on ethnicity, we found the benefits of kidney transplantation among Asians, but not among Caucasians. Causes of ED in kidney transplant recipients are multifactorial (27). We guess that the characteristics of kidney transplantation groups in different regions of the world may be one reason for the racial differences. In addition, the insufficiency of the sample size of these enrolled studies may have led to a certain deviation in racial differences.

We did a subgroup analysis based on different tools, and found that the improvement of erectile function in the kidney transplantation group was more significant when IIEF-5 was utilized. As an abridged version of IIEF-15, the IIEF-5 scale possesses favorable properties for detecting the presence and severity of ED (28). Also, items in the IIEF-5 are phrased to reference the prior six-month period, which conforms with the NIH's current reference period for establishing a diagnosis of ED. By contrast, items in the erectile function domain of IIEF-15 make a reference period of four weeks (28). Therefore, it may relate to the results of the subgroup analysis. In addition, the small sample size may also cause some deviation in the results. Van Kollenburg et al. studied the stability and validity of the electronic versions of IIEF-5 and IIEF-15 and found that both had excellent internal consistency and test-retest reliability (29).

As confirmed by clinical studies, the IIEF-15 questionnaire is a validated psychometric tool, which serves to assess the different sexual function domains (30). Our study found the IIEF domain score, which reflects the psychological aspects (IIEF-SD) was improved significantly in the kidney transplantation group, except the orgasmic function and patient satisfaction (IIEF-OF, IIEF-IS, and IIEF-OS). There was no improvement in intercourse satisfaction and overall satisfaction, which may suggest that the improvement of erectile function may be limited because a primary outcome of ED for most patients is the inability to attain satisfactory sexual intercourse (30). Likewise, Mota thought body image satisfaction after undergoing kidney transplantation is associated with erectile function, with better perceptions being associated with better erectile response (31). One Limitation of the IIEF scale is the absence of evaluation of the partner relationship. Tavallaii et al. (25) compared the sexual function of male renal transplantation recipients and hemodialysis patients using the Relationship and Sexuality Scale (RSS). They found renal transplantation patients in comparison with the hemodialysis group reported a better overall sexual relationship, and all subscales including sexual function, sexual frequency, and sexual fear. Also, kidney recipients had significantly more sexual intercourse in 2 weeks before the study.

In addition, we found that the improvement of erectile function was not significant in a sub-analysis on the studies with pre- and post- transplanted patients. In the studies we included, most of the prospective studies were followed up for only 6 months, which may lead to no meaningful benefits detected after kidney transplantation. 
Even so, it is worth taking note that a large percentage of kidney transplant patients still have not improved their erectile function. This may be related to age, use of immunosuppressants, and other comorbidities (32).

Multiple hormonal abnormalities have been observed in patients with ESRD $(33,34)$. Published studies report low circulating testosterone levels, abnormalities in Leydig cell morphology with spermatogenic maturation arrest, and variable levels of FSH and $\mathrm{LH}(14,35)$. According to Carrero et al. (36), definite testosterone deficiency occurs among about $44 \%$, testosterone insufficiency, about $33 \%$, and normal testosterone level, only $23 \%$, among patients with ESRD. Even with regular hemodialysis, abnormalities of the hypothalamic-pituitary-testicular axis persist $(37,38)$. And whether kidney transplantation could reverse the alterations induced in the pituitary-testicular axis is still uncertain.

Several of the studies we included reported changes in hormone levels. Our meta-analysis of these studies showed that serum testosterone level was increased, but serum PRL and LH levels were reduced in the renal transplantation group. No significant difference was noted in serum FSH level between the two groups. Previous studies suggested that the increased mean testosterone with suppressed LH and restored serum PRL levels indicate Leydig cell function recovery after kidney transplantation (39). Testosterone plays an important part in erectile function in males $(40,41)$. A meta-analysis of 14 randomized controlled studies, including 2,298 men, suggested that testosterone therapy improved the IIEF erectile function domain score by 2.31 when compared to placebo (42). Therefore, an increase in serum testosterone level after kidney transplantation may have a role in improving erectile function. Corona found that elevated PRL could lead to decreasing libido (43). Therefore, the increased sexual desire in the kidney transplantation group found by our study may be related to the decreased serum PRL level. In recent years, more and more researchers have proposed that there is an endocrinological basis for sexual dysfunction in ESRD, and kidney transplantation can improve sexual function by correcting the abnormalities of the hypothalamus-pituitarygonadal axis. However, it also remains to be proven by large sample studies whether a long-term functional kidney transplantation graft could bring hormone levels back to normal.

Although we searched the databases as many as possible to capture comprehensive literature and got relatively optimistic results, there are some limitations in our study.
Firstly, heterogeneities were observed in some outcomes of our study, and the reasons for heterogeneities were complicated. Heterogeneity may be attributed to different study designs and patient characteristics (e.g., age, dialysis duration, etiology of renal failure), type of intervention (e.g., surgical procedure for kidney transplantation), follow-up time, study quality and sample size. Nevertheless, we were not in a position to perform all of these subgroup analyses because of the limited data available. Secondly, considering that most of the studies we included were observational studies, our study could not prove causality and unmeasured confounding factors could not be eliminated. Finally, the sample size of this meta-analysis was not large enough, which suggested that further prospective studies of larger samples were needed.

In summary, kidney transplantation may be associated with improved erectile function in patients with ESRD. This may be attributable to the correction of endocrine hormone disorders in patients after kidney transplantation, which was manifested by an increase in serum testosterone and a decrease in LH and PRL levels. More studies, especially large prospective ones, are required to validate our results in the future.

\section{Acknowledgments}

Thanks to Liu Chao for her long-term care of the first author of this article.

Funding: This study was financially supported by Zhao YiCheng Medical Science Foundation (ZYYFY2018031).

\section{Footnote}

Reporting Checklist: The authors have completed the PRISMA reporting checklist. Available at http://dx.doi. org/10.21037/tau-20-604

Peer Review File: Available at http://dx.doi.org/10.21037/ tau-20-604

Conflicts of Interest: All authors have completed the ICMJE uniform disclosure form (available at http://dx.doi. org/10.21037/tau-20-604). The authors have no conflicts of interest to declare.

Etbical Statement: The authors are accountable for all aspects of the work in ensuring that questions related to the accuracy or integrity of any part of the work are 
appropriately investigated and resolved.

Open Access Statement: This is an Open Access article distributed in accordance with the Creative Commons Attribution-NonCommercial-NoDerivs 4.0 International License (CC BY-NC-ND 4.0), which permits the noncommercial replication and distribution of the article with the strict proviso that no changes or edits are made and the original work is properly cited (including links to both the formal publication through the relevant DOI and the license). See: https://creativecommons.org/licenses/by-nc-nd/4.0/.

\section{References}

1. NIH Consensus Conference. Impotence. NIH Consensus Development Panel on Impotence. JAMA 1993;270:83-90.

2. Salonia A, Castagna G, Saccà A, et al. Is erectile dysfunction a reliable proxy of general male health status? The case for the International Index of Erectile FunctionErectile Function domain. J Sex Med 2012;9:2708-15.

3. Fiuk JV, Tadros NN. Erectile dysfunction in renal failure and transplant patients. Transl Androl Urol 2019;8:155-63.

4. Esen B, Kahvecioglu S, Atay AE, et al. Evaluation of relationship between sexual functions, depression and quality of life in patients with chronic kidney disease at predialysis stage. Renal failure 2015;37:262-7.

5. Narang GL, Pannell SC, Laviana AA, et al. Patientreported outcome measures in urology. Curr Opin Urol 2017;27:366-74.

6. Stang A. Critical evaluation of the Newcastle-Ottawa scale for the assessment of the quality of nonrandomized studies in meta-analyses. Eur J Epidemiol 2010;25:603-5.

7. El-Bahnasawy MS, El-Assmy A, Dawood A, et al. Effect of the use of internal iliac artery for renal transplantation on penile vascularity and erectile function: a prospective study. J Urol 2004;172:2335-9.

8. Mehrsai A, Mousavi S, Nikoobakht M, et al. Improvement of erectile dysfunction after kidney transplantation: the role of the associated factors. Urol J 2006;3:240-4.

9. Peng T, Zhang GT, Chen M, et al. Erectile function in male kidney transplant recipients and effects of different methods of renal arterial anastomosis. Zhonghua Nan Ke Xue 2007;13:396-9.

10. Mirone V, Longo N, Fusco F, et al. Renal Transplantation Does Not Improve Erectile Function in Hemodialysed Patients. Eur Urol 2009;56:1047-53.

11. Al Khallaf HH. Analysis of sexual functions in male nondiabetic hemodialysis patients and renal transplant recipients. Transpl Int 2010;23:176-81.

12. Ji ZG, Tian Y, Tang YW, et al. Influence of the interruption of arteria iliaca interna distal end on penile vascularity and erectile function in male renal transplant recipients. Zhonghua Nan Ke Xue 2010;16:341-4.

13. Teng LC, Wang CX, Chen L. Improved erectile function and sex hormone profiles in male Chinese recipients of kidney transplantation. Clin Transplant 2011;25:265-9.

14. Park MG, Koo HS, Lee B. Characteristics of testosterone deficiency syndrome in men with chronic kidney disease and male renal transplant recipients: A cross-sectional study. Transplant Proc 2013;45:2970-4.

15. Yavuz D, Acar FN, Yavuz R, et al. Male sexual function in patients receiving different types of renal replacement therapy. Transplant Proc 2013;45:3494-7.

16. Hart A, Smith JM, Skeans MA, et al. OPTN/SRTR 2016 Annual Data Report: Kidney. Am J Transplant 2018;18 Suppl 1.

17. Navaneethan SD, Vecchio M, Johnson DW, et al. Prevalence and correlates of self-reported sexual dysfunction in CKD: a meta-analysis of observational studies. Am J Kidney Dis 2010;56:670-85.

18. Levy NB. Sexual adjustment to maintenance hemodialysis and renal transplantation: national survey by questionnaire: preliminary report. Trans Am Soc Artif Intern Organs 1973;19:138-43.

19. Nassir A. Erectile dysfunction risk factors for patients entering dialysis programme. Andrologia 2010;42:41-7.

20. Lai CF, Wang YT, Hung KY, et al. Sexual dysfunction in peritoneal dialysis patients. Am J Nephrol 2007;27:615-21.

21. Krishnan R, Izatt S, Bargman JM, et al. Prevalence and determinants of erectile dysfunction in patients on peritoneal dialysis. Int Urol Nephrol 2003;35:553-6.

22. Pourmand G, Emamzadeh A, Moosavi S, et al. Does renal transplantation improve erectile dysfunction in hemodialysed patients? What is the role of associated factors? Transplant Proc 2007;39:1029-32.

23. Lundy SD, Vij SC. Male infertility in renal failure and transplantation. Transl Androl Urol 2019;8:173-81.

24. Papadopoulou E, Varouktsi A, Lazaridis A, et al. Erectile dysfunction in chronic kidney disease: From pathophysiology to management. World J Nephrol 2015;4:379-87.

25. Tavallaii SA, Mirzamani M, Heshmatzade Behzadi A, et al. Sexual function: a comparison between male renal transplant recipients and hemodialysis patients. J Sex Med 2009;6:142-8.

26. Ahmad M, Rafiuddin Q, Hassan U, et al. Impact of renal 
transplantation on erectile dysfunction due to chronic renal failure in male patients. J Ayub Med Coll Abbottabad 2009;21:69-71.

27. El-Assmy A. Erectile dysfunction in hemodialysis: A systematic review. World J Nephrol 2012;1:160-5.

28. Rosen RC, Cappelleri JC, Smith MD, et al. Development and evaluation of an abridged, 5-item version of the International Index of Erectile Function (IIEF-5) as a diagnostic tool for erectile dysfunction. Int J Impot Res 1999;11:319-26.

29. van Kollenburg RAA, de Bruin DM, Wijkstra H. Validation of the Electronic Version of the International Index of Erectile Function (IIEF-5 and IIEF-15): A Crossover Study. J Med Internet Res 2019;21:e13490.

30. Rosen RC, Riley A, Wagner G, et al. The international index of erectile function (IIEF): a multidimensional scale for assessment of erectile dysfunction. Urology 1997;49:822-30.

31. Mota RL, Fonseca R, Santos JC, et al. Sexual Dysfunction and Satisfaction in Kidney Transplant Patients. J Sex Med 2019;16:1018-28.

32. Pertuz W, Castaneda DA, Rincon O, et al. Sexual dysfunction in patients with chronic renal disease: does it improve with renal transplantation? Transplant Proc 2014;46:3021-6.

33. Lim VS, Fang VS. Gonadal dysfunction in uremic men. A study of the hypothalamo-pituitary-testicular axis before and after renal transplantation. Am J Med 1975;58:655-62.

34. Gómez F, de la Cueva R, Wauters JP, et al. Endocrine abnormalities in patients undergoing long-term hemodialysis. The role of prolactin. Am J Med

Cite this article as: Kang J, Tian J, Lu Y, Song Y, Liu X. Erectile function after kidney transplantation: a meta-analysis. Transl Androl Urol 2020;9(5):1967-1979. doi:10.21037/tau-20-604
1980;68:522-30.

35. Stewart-Bentley M, Gans D, Horton R. Regulation of gonadal function in uremia. Metabolism 1974;23:1065-72.

36. Carrero JJ, Qureshi AR, Nakashima A, et al. Prevalence and clinical implications of testosterone deficiency in men with end-stage renal disease. Nephrol Dial Transplant 2011;26:184-90.

37. Foulks CJ, Cushner HM. Sexual dysfunction in the male dialysis patient: pathogenesis, evaluation, and therapy. Am J Kidney Dis 1986;8:211-22.

38. Holdsworth S, Atkins RC, de Kretser DM. The pituitarytesticular axis in men with chronic renal failure. $\mathrm{N}$ Engl J Med 1977;296:1245-9.

39. Yadav R, Mehta SN, Kumar A, et al. A prospective analysis of testicular androgenic function in recipients of a renal allograft. Int Urol Nephrol 2008;40:397-403.

40. Snyder PJ, Bhasin S, Cunningham GR, et al. Effects of Testosterone Treatment in Older Men. N Engl J Med 2016;374:611-24.

41. Almehmadi Y, Yassin AA, Nettleship JE, et al. Testosterone replacement therapy improves the health-related quality of life of men diagnosed with late-onset hypogonadism. Arab J Urol 2016;14:31-6.

42. Corona G, Rastrelli G, Morgentaler A, et al. Meta-analysis of Results of Testosterone Therapy on Sexual Function Based on International Index of Erectile Function Scores. Eur Urol 2017;72:1000-11.

43. Corona G, Isidori AM, Aversa A, et al. Endocrinologic Control of Men's Sexual Desire and Arousal/Erection. J Sex Med 2016;13:317-37. 
Supplementary

Table S1 The Newcastle-Ottawa Scale scores for prospective studies

\begin{tabular}{|c|c|c|c|c|c|c|c|c|c|}
\hline Study & \multicolumn{4}{|c|}{ Selection } & Comparability & \multicolumn{3}{|c|}{ Outcomes } & $\begin{array}{l}\text { Total } \\
\text { scores }\end{array}$ \\
\hline Mehrsai (2006) & 1 & 1 & 1 & 1 & 2 & 0 & 0 & 1 & 7 \\
\hline Peng (2007) & 1 & 1 & 1 & 1 & 2 & 0 & 0 & 1 & 7 \\
\hline Mirone (2009) & 1 & 1 & 1 & 1 & 2 & 0 & 0 & 1 & 7 \\
\hline Jl (2010) & 1 & 1 & 1 & 1 & 2 & 0 & 0 & 1 & 7 \\
\hline
\end{tabular}

Table S2 The Agency for Healthcare Research and Quality (AHRQ) for cross-sectional studies

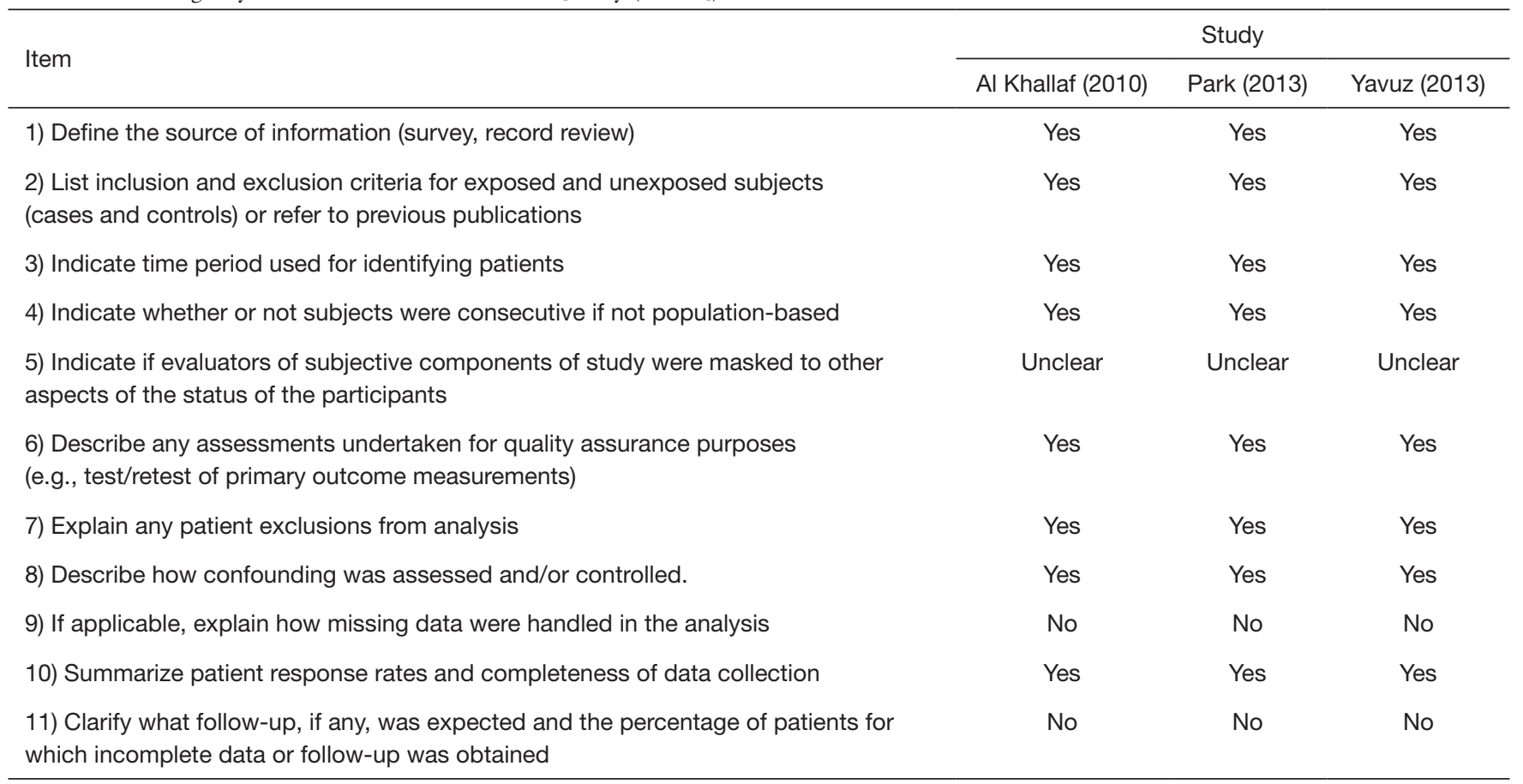

